\title{
LOADING SURFACE IN THE PLASTIC AND CREEP STRAINING COUPLED WITH DIRECT CURRENT
}

\author{
Andrew Rusinko, Peter Varga \\ Obuda University, Hungary \\ e-mail:ruszinko.endre@bgk.uni-obuda.hu
}

\begin{abstract}
The paper is aimed to give a visual representation of the loading surface for different types of irrecoverable deformation in the electrical field. Three situations of deforming coupled with direct current (DC) are discussed: primary creep, secondary creep, and plastic deformation. Understanding the evolution of loading surface under the action of current is considered to be the necessary step pertaining to design forming processes. Therefore, the analysis of the evolution of loading surface in the electrical field and its comparison with the case of ordinary loading is the main subject of this paper.
\end{abstract}

Keywords: direct current, plastic deformation, creep deformation, synthetic theory

\section{Introduction}

Three key concepts form the basis of almost all theories of plasticity. They are:

- Yield criteria, which predict whether the solid responds elastically or plastically. The criteria define which combination of multi-axial stress will cause yielding and describe the surface in the stress space that demarks the boundary between the elastic and plastic behavior of materials. Development of yielding criteria is hence pivotal in predicting whether or not a material will begin to yield under a given stress state.

- Strain hardening rules, which control the way in which resistance to plastic flow increases with plastic straining. The hardening rule gives the evolution in the yield criterion during plastic deformation. Four typologies of hardening may arise during irrecoverable straining: (i) isotropic hardening, which refers to the proportional expansion of the initial yield surface; (ii) kinematic hardening, if the deforming material shows a yield surface that does not change in form and size but translates in the stress space; (iii) rotational hardening, which causes the yield locus to rotate; (iv) distortional hardening, which causes the yield locus to distort. Stress states inside the yield/loading surface (subsequent yield surface) result in elastic deformation. The material yields when the stress state reaches the yield surface and further loading causes plastic deformation. Stresses outside the loading surface do not exist, and the plastic strain and shape of the loading surface evolve to maintain stresses either inside or on the loading surface.

- The plastic flow rule, which determines the relationship between stress and plastic strain under multi-axial loading.

These concepts will be considered in terms of the synthetic theory of irrecoverable deformation (Rusinko and Rusinko, 2009, 2011) for the case of ordinary loading and that coupled with direct current. With DC, the following phenomena are recorded and will be discussed (Andrawes et al., 2007; Chen and Yang, 2008; Kinney et al., 2009; Li et al., 2015; Perkins et al., 2007; Ross et al., 2007; Sanmartin et al., 1983; Nguyen et al., 2016; Yang and Zhao, 2010; Zhao et al., 2012, 2016; Zhao and Yang, 2014): 
(i) an increase in the steady state creep rate coupled with DC;

(ii) an increase in transient creep and shortening of its duration under the action of the current;

(iii) stress-drop during plastic deformation with a pulsed electric current.

Our first steps to model the phenomena listed (schematically sketched in Fig. 1) can be found in Rusinko (2016) and Rusinko and Varga (2018), which show good agreement with experimental results. The main goal of this paper is (a) to strengthen the capacity of the results obtained earlier - the formulae proposed by Rusinko and Varga (2018) give satisfactory results for the steady state creep as a function of current intensity only for one temperature $(323 \mathrm{~K})$, (b) to give a visual representation of the evolution of loading surface for irrecoverable strains in the electrical field.

(a)

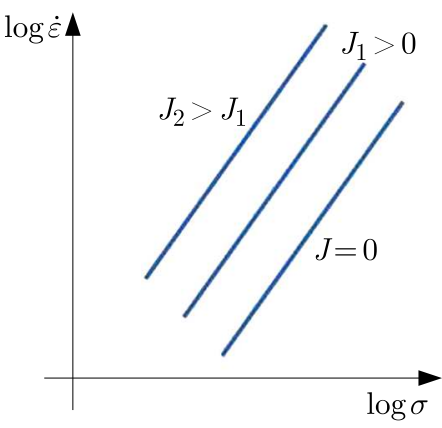

(b)

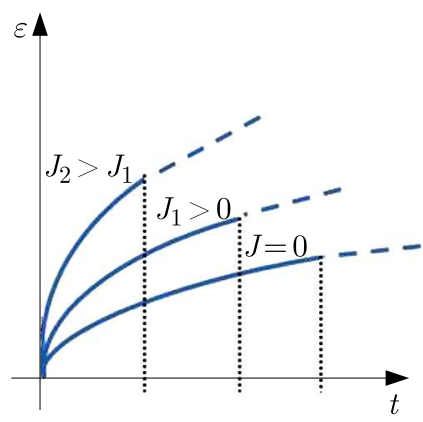

(c)

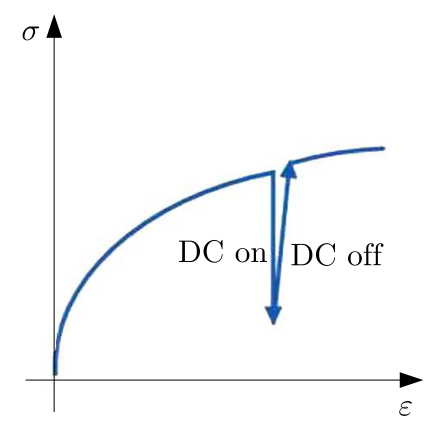

Fig. 1. The effect of DC upon (a) steady state creep rate, (b) transient creep, (c) plastic straining

\section{Synthetic theory}

The synthetic theory incorporates the Budiansky slip concept and the Sandres flow theory.

As in the Budiansky concept, irrecoverable deformation at a point of the body is calculated at two levels of the material structure: micro- and macro-level. For the macro-level, we take as the elementary volume of the body $V$, which consists of a large quantity of microvolumes $V_{0}$ (slip systems), each being an element of the continuous body, capable of deforming under the applied forces via slip of one part of $V_{0}$ in relation to another. Accordingly to Batdorf and Budiansky (1949), it is assumed that the stress state in every slip system is the same as the macrostress-state. Nevertheless, in contrast to the even distribution of the stress over slip systems, the plastic deformation (or plastic slip) within $V_{0}$ strongly depends on the orientation of the slip system relative to the direction of the acting forces. It is assumed that the quantity of $V_{0}$ is so great (theoretically it tends to infinity) that every possible orientation of slip systems exists at the point of the body. The total deformation of $V$ is determined as a sum of the components of deformations generated in volumes $V_{0}$.

The realization of Budiansky's approach to the modelling of irrecoverable deformation takes place in the three-dimensional subspace $\mathcal{S}^{3}$ of the five-dimensional Ilyushin deviatoric space, where the loading is presented by a stress vector $\mathbf{S}$ whose length equals to the effective stress $\tau_{0}$. The yield criterion is taken as the von Mises rule, which gives a sphere of radius $\sqrt{2 / 3} \sigma_{S}$ in $\mathcal{S}^{3}$ ( $\sigma_{S}$ is the yield limit of the material)

$$
S_{1}^{2}+S_{2}^{2}+S_{3}^{2}=\frac{2}{3} \sigma_{S}^{2}
$$

The specific feature of the synthetic theory consists mainly in formulation of the hardening rule. Let us draw tangent planes at every point of sphere (2.1). As a result, we obtain the system 
of equidistant planes. The position of the plane in $\mathcal{S}^{3}$ is given by the normal vector $\mathbf{N}$ and its distance from the origin of coordinates $H_{N}$. It is clear that in the virgin state, $H_{N}=\sqrt{2 / 3} \sigma_{S}$ for all directions. Plastic deformation starts when the stress vector reaches sphere (2.1), or in other words, the first plane is on the endpoint of $\mathbf{S},|\mathbf{S}|=\sqrt{2 / 3} \sigma_{S}$.

Following Sanders (1954), we treat the yield surface as the inner envelope of the tangent planes (Fig. 2a). To extend this principle for the further increase in loading as well $|\mathbf{S}|>\sqrt{2 / 3} \sigma_{S}$, we suggest that the stress vector translates on its endpoint (loading point) those planes which it reaches. Now, the loading surface, being the inner envelope of tangent planes, takes the form as shown in Fig. 2b. Therefore, we do not define in advance the evolution of the loading surface during plastic straining, but the loading surface follows the planes displaced by the stress vector so that be their inner envelope.
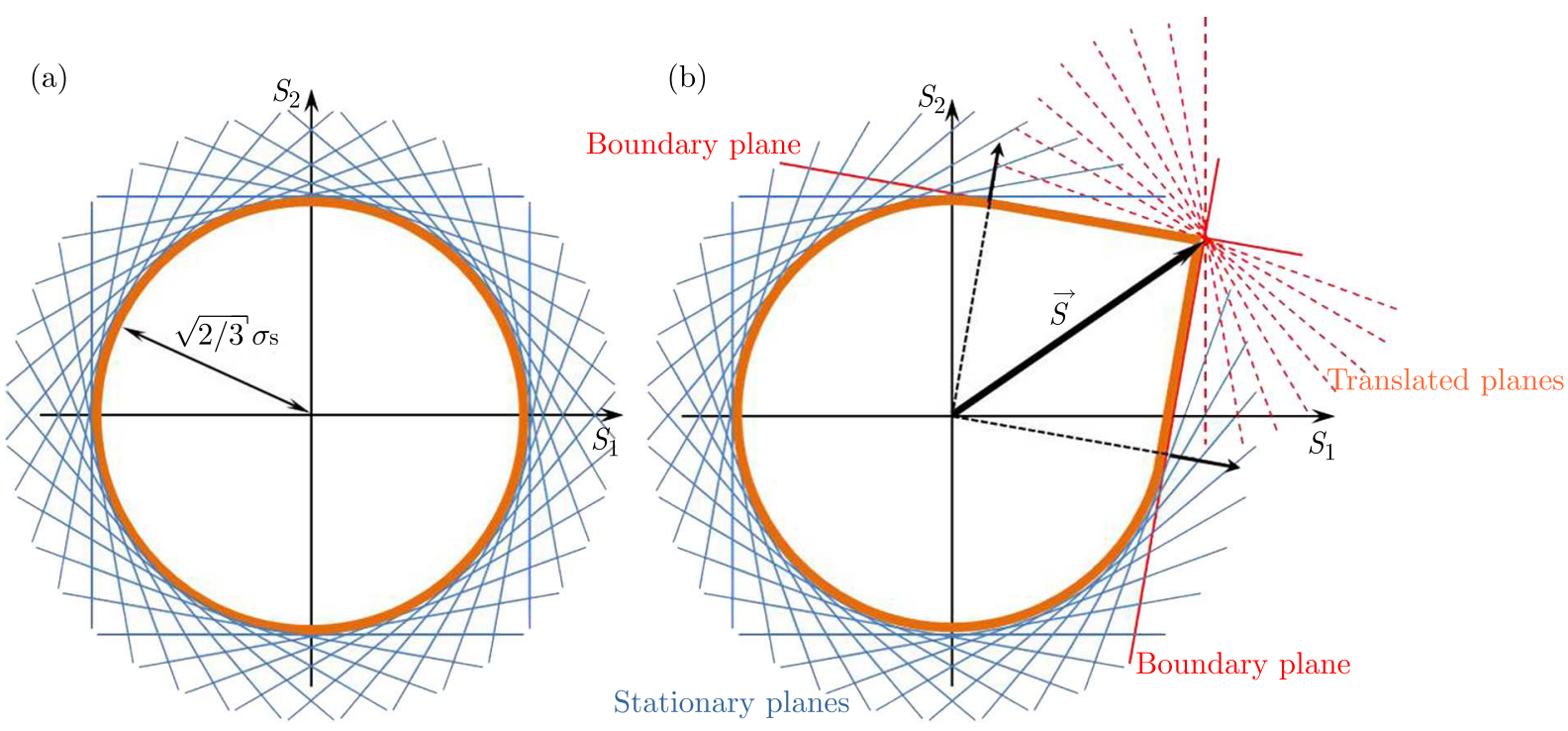

Fig. 2. The interpretation of (a) yield- and (b) loading surface as the inner envelope of tangent planes

The physical sense of plane movements is that each plane translated by $\mathbf{S}$ corresponds to plastic slip within appropriate $V_{0}$. For example, in uniaxial tension, the first plane which is at the endpoint of the stress vector corresponds to the slip system inclined $45^{\circ}$ with respect to the acting stress. As the loading increases, other planes are shifted by the stress vector, which means that slip systems with less favorable orientations are involved in the progress of plastic deformation. Micro-deformation developed by motion of one plane, i.e. within one slip system, is modelled by an elementary strain vector which is assumed to be perpendicular to the plane (Fig. 3).

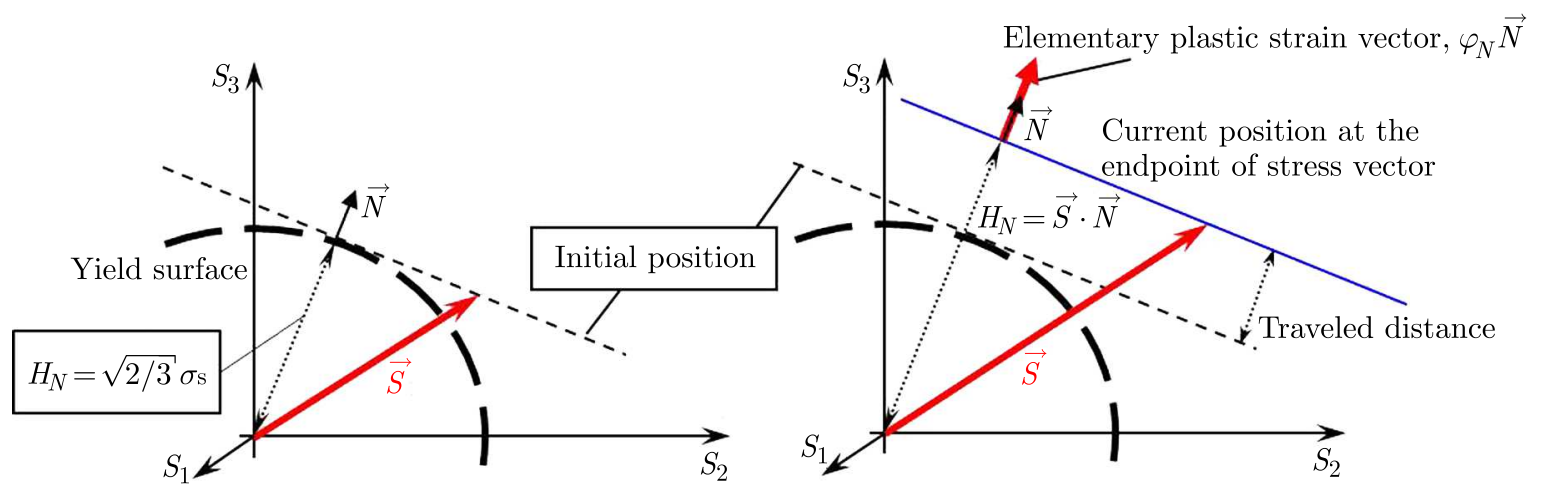

Fig. 3. The formulation of the flow rule in terms of the synthetic theory 
It becomes obvious that the plane distance $H_{N}$ reflects the measure of the hardening of material. Indeed, the greater distance to a plane, the greater stress vector is needed to reach it and induce plastic deformation.

The total level of strains at a point of body, strain vector $\mathbf{e}$, is determined by adding plastic strains developed at every moving plane (Rusinko and Rusinko, 2009, 2011)

$$
\mathbf{e}=\iiint_{V} \varphi_{N} \mathbf{N} d V
$$

where $\varphi_{N}$ is referred to as strain intensity and symbolizes an average measure of plastic deformation within one slip system. The strain intensity is determined from the constitutive equation of the synthetic theory

$$
d \psi_{N}=r d \varphi_{N}-K \psi_{N} d t
$$

where $\psi_{N}$ is defect intensity, an average measure of defects in the crystalline grid (dislocations, point defects, twins, etc.) arising during irrecoverable straining and hindering its progress. $\psi_{N}$ is defined as

$$
\psi_{N}=H_{N}^{2}-I_{N}^{2}-S_{P}^{2}
$$

and

$$
\psi_{N}= \begin{cases}(\mathbf{S} \cdot \mathbf{N})^{2}-I_{N}^{2}-S_{P}^{2} & \text { plane is at the enpoint of } \mathbf{S}, H_{N}=\mathbf{S} \cdot \mathbf{N} \\ 0 & \text { vector } \mathbf{S} \text { does not reach a plane, } H_{N}>\mathbf{S} \cdot \mathbf{N}\end{cases}
$$

The scalar product $\mathbf{S} \cdot \mathbf{N}$ gives the resolved shear stress acting with the slip system. $S_{P}$ denotes the radius of the von Mises sphere.

Equations (2.3) and (2.4) form the flow rule for every slip system (microdeformation), and Eq. (2.2) gives an average of slip flows (macrodeformation). Equation (2.3) says that the increment in defects is a result of two competing processes: i) development of plastic (instant) deformation and ii) time-dependent relaxation of the defects. Concrete loading and thermal circumstances determine which mechanism dominates. Such a formulation covers problems ranged from plastic strain to unsteady/steady creep, as well as the relaxation of defects.

Equation (2.4) states that the greater the distance traveled by a plane on the endpoint of $\mathbf{S}$ (see Fig. 2), the greater is the number of defects nucleated and cumulated in the process of plastic deforming.

The rate-dependent fashion of plastic flow is provided by the function $I_{N}$, the rate integral, in formula $(2.4)$

$$
I_{N}(t)=B \int_{0}^{t} \dot{\mathbf{S}} \cdot \mathbf{N} \exp [-p(t-s)] d s
$$

which governs:

- the value of yield limit as a function of the loading rate $\dot{\mathbf{S}}$ (we use the notion of "creep limit" $\sigma_{P}$, as $\dot{\mathbf{S}}=0$, i.e. when creep deformation is considered) and

- the kinetics of primary creep.

The rate-integral serves as a measure of the difference between the average stress acting in material and the local stresses (stresses of the third kind). This difference results in elastic distortions of the crystalline lattice, which increases with the loading rate and hinders the progress of plastic 
deformation. At the same time, the distortions can relax at favorable conditions, e.g. high temperature and/or constant in time stresses. This process facilitates the motion of dislocations and acts as a driving force of primary creep. As the energy cumulated in active loading is exhausted, the material deforms in the regime when softening and hardening are in balance. Summarizing, the $I_{N}(t)$ increases as $\dot{\mathbf{S}}>0$ and decreases under subsequent constant stresses (primary creep). The condition $I_{N}(t) \rightarrow 0$ symbolizes the transition to the secondary creep.

The function $K$ in Eq. (2.4), which regulates the steady state creep rate, is defined as

$$
K=K_{1}(T) K_{2}\left(\tau_{0}\right) \quad K_{1}=\exp \left(-\frac{Q}{R T}\right) \quad K_{2}=\frac{9 \sqrt{3} c r}{2 \sqrt{2} \pi} \tau_{0}^{k-2}
$$

In formulae (2.3), (2.6) and (2.7), $c, k, B$ and $p$ are the model constants to be determined to achieve the best fit between the analytical and experimental results.

Based on the above, the main features of the synthetic theory are summarized as follows:

- While a material in its virgin state is assumed to possess isotropic plastic properties, sphere (2.1), the plastic deformation is of definitely anisotropic fashion. The strain hardening of the material varies depending on the position of tangent planes, i.e. the orientation of the slip system.

- The evolution of the loading surface is not prescribed a priori, but strongly depends on the hodograph of the stress vector, i.e. the loading path governs entirely the transformation of the loading surface.

- At the loading point, a corner singularity arises on the loading surface, which is of crucial importance when curvilinear loading trajectories are considered.

The flow rule of the synthetic theory is defined in such a way that all types of deformation, plastic or creep, can be described from the same system of constitutive equations (2.2)-(2.6). This is due to the fact that the synthetic theory is of physical nature, irrecoverable deformation $\varphi_{N}$ is related to the function which reflects the amount of crystalline grid defects nucleated and multiplied during the deformation $\psi_{N}$. Further, the time- and loading-rate-dependent nature of the rate integral, together with Eq. (2.4), completes the formulation of irrecoverable deformation from the unified position. Further throughout, we use the single notion of irrecoverable deformation, whose components, instant or time-dependent, manifest themselves depending on the loading and thermal regimes.

\section{Synthetic theory extended to the case of deformation in an electrical field}

To model the effect of DC upon irrecoverable deformation we address the researchers' report about what action the electron wind exerts upon plastic or creep straining. This action can be summarized as follows:

- DC-induced Joule heating causing a change in local temperature and resulting in time-dependent plastic deformation,

- The momentum exchange between moving electrons and lattice atoms reducing the energy barrier and increasing the migration velocity of atoms,

- The intensification of the current field assisted sliding rate and diffusional creep.

To reflect the points listed above, we enhance the action of resolved shear $\left(\mathbf{S} \cdot \mathbf{N}=H_{N}\right)$ by introducing a term that expresses the assisting role of passing current

$$
\psi_{N}=H_{N}^{2}\left(1+C^{2}\right)-\left(I_{N}^{C}\right)^{2}-S_{P}^{2}
$$


where $C=C(J, T), J$ is current intensity $\left[\mathrm{kA} / \mathrm{cm}^{2}\right]$. In other words, Eq. (3.1) expresses the intensification of slides within a slip system due to the passage of current. The function $C$ is defined as a product of two functions

$$
C=U(J) W(T)
$$

both related to their arguments as power functions

$$
U=u_{1} J^{u_{2}} \quad W=\left(w_{1} T-w_{2}\right)^{w_{3}}+w_{4}
$$

where $u_{k}$ and $w_{l}$ are model constants to be determined to best fit the experiments. It is the idea to define function $C$ via Eqs. (3.2) and (3.3) that makes it possible to obtain satisfactory results for $\dot{\varepsilon} \sim J^{2}$ plots for a wide range of temperatures (see below Fig. $7 \mathrm{~b}$ ).

To catch the effect of DC upon the parameters of primary creep we propose to modify $I_{N}$ as

$$
I_{N}^{C}=B_{C} \int_{0}^{t} \frac{d \mathbf{S}}{d s} \mathbf{N} \exp \left[-p_{C}(t-s)\right] d s \quad B_{C}=B+B_{1} J^{B_{2}} \quad p_{C}=p+p_{1} J^{p_{2}}
$$

To account for the DC-induced Joule heating, we introduce term 5.23J $J^{2}$ (Zhao and Yang, 2014) into formula (2.7) for $K_{1}(T)$

$$
K_{1}(T)=\exp \left[-\frac{Q}{R\left(T+5.23 J^{2}\right)}\right]
$$

\section{Plastic deformation: ordinary case and deformation under DC impulse}

Consider uniaxial tension with the sole non-zero stress tensor component $\sigma$, when the stress vector lies along $S_{1}$-axis, $\mathbf{S}(\sqrt{2 / 3} \sigma, 0,0)$. The orientation of tangent planes is given by the components of the unit vector $\mathbf{N}^{1}$

$$
N_{1}=\cos \alpha \cos \beta \cos \lambda \quad N_{2}=\sin \alpha \cos \beta \cos \lambda \quad N_{3}=\sin \beta \cos \lambda
$$

Now, the distance to the plane, which is at the endpoint of $\mathbf{S}$, is

$$
H_{N}=S_{1} N_{1}+S_{2} N_{2}+S_{3} N_{3}=\sqrt{\frac{2}{3}} \sigma \cos \alpha \cos \beta \cos \lambda
$$

It is clear that the first plane reached by the $\mathbf{S}$ on sphere (2.1) is perpendicular to $S_{1}$-axis, $\alpha, \beta, \lambda=0$, which means the most favorably oriented slip system. Further growth in $|\mathbf{S}|$ increases the set of planes shifted by $\mathbf{S}$.

If to write down Eq. (2.3) as

$$
r \varphi_{N}=\psi_{N}+K \int \psi_{N} d t
$$

we can ignore the time-integral due to the small duration of plastic deforming. As a result, we get

$$
r \varphi_{N}=\psi_{N}
$$

It says that plastic deformation is accompanied by the nucleation and multiplication of defects/obstacles, and its development demands a constant increase in acting stresses.

\footnotetext{
${ }^{1}$ Angle $\lambda$ gives the position of the plane in $\mathcal{S}^{3}$ which is the trace of the plane tangential to the five-dimensional yield surface.
} 
Therefore, within one slip system, Eqs. (2.5) and (4.4) give the strain intensity $\mathrm{as}^{2}$

$$
\varphi_{N}=\frac{1}{r}\left(H_{N}^{2}-\sigma_{S}^{2}\right)=\frac{2}{3 r}\left[(\sigma \cos \alpha \cos \beta \cos \lambda)^{2}-\sigma_{S}^{2}\right]
$$

The macrodeformation is determined from Eq. (2.2)

$$
e=\frac{2}{3 r} \int_{-\alpha_{1}}^{\alpha_{1}} \int_{-\beta_{1}}^{\beta_{1}} \int_{0}^{\lambda_{1}}\left[(\sigma \cos \alpha \cos \beta \cos \lambda)^{2}-\sigma_{S}^{2}\right] \cos \alpha \cos ^{2} \beta \cos \lambda d \alpha d \beta d \lambda
$$

The boundaries of integration, i.e. the range of angles where $\varphi_{N}$ is nonzero, are

$$
\cos \lambda_{1}=\frac{\sigma_{S}}{\sigma \cos \alpha \cos \beta} \quad \cos \alpha_{1}=\frac{\sigma_{S}}{\sigma \cos \beta} \quad \cos \beta_{1}=\frac{\sigma_{S}}{\sigma}
$$

By integrating in (4.6) within range (4.7), we obtain

$$
\begin{aligned}
& e=a F(b) \quad a=\frac{\pi \sigma_{S}^{2}}{9 r}=\text { const } \quad b=\cos \beta_{1} \\
& F(b)=\frac{1}{b^{2}}\left(2 \sqrt{1-b^{2}}-5 b^{2} \sqrt{1-b^{2}}+3 b^{4} \ln \frac{1+\sqrt{1-b^{2}}}{b}\right)
\end{aligned}
$$

$F(b)$ is a decreasing function of $b$, i.e. an increasing one of $\sigma$. To simplify the calculations, we approximate the function $F$ from (4.8) as

$$
F \approx\left(\frac{1}{x}-1\right)^{2} \quad F(1)=F^{\prime}(1)=0
$$

Experiments conducted by Nguyen with co-workers (Nguyen et al., 2016) show that a short electric impulse results in an immediate decrease in the applied stress (Fig. 1c). In other words, the tensile stress needed to maintain the same deformation as before the impulse, incurs a step-wise decrease (the portions following the stress-drop are not considered here). The flow stress decreases with the increasing current for two reasons: (i) the corresponding temperature due to Joule heating increases as the current increases; (ii) the electric current helps the glide and climb of dislocation, and the decrease of dislocation density and dislocation multiplication rate promotes the reduction of flow stress.

Once a pulse of current is "On", Eqs. (3.1) and (4.4) give

$$
\varphi_{N C}=\frac{1}{r}\left[H_{N C}^{2}\left(1+C^{2}\right)-\sigma_{S}^{2}\right]
$$

To maintain the deformation at the same level as before the impulse, we demand that

$$
\varphi_{N C}=\varphi_{N}
$$

which, on the base of (4.5) and (4.10), can be ensured only if

$$
H_{N C}^{2}\left(1+C^{2}\right)=H_{N}^{2} \Longrightarrow H_{N C}=\frac{H_{N}}{\sqrt{1+C^{2}}}
$$

The relation between $H_{N C}$ and $H_{N}$ means that the electric pulse results in jump-wise motions of planes toward the origin of coordinates. Since the ratio $H_{N C} / H_{N}$ does not depend on the

\footnotetext{
${ }^{2}$ If to consider plastic deformation at low homology temperatures, the role of the rate-integral diminishes.
} 
angles $\alpha, \beta$ and $\lambda$, we conclude that the plane displacements are of the same magnitude in all directions. This implies that the loading surface, at the instant of the electric pulse, preserves its shape as before the action of current: see Fig. 4a. The length of $\mathbf{S}_{C}$ vector, i.e. the value of tension stress $\sigma_{C}$, is calculated via Eq. (4.12) at $\alpha, \beta, \lambda=0$ as

$$
\sigma_{C}=\frac{\sigma}{\sqrt{1+C^{2}}}
$$

Figure $4 \mathrm{~b}$ demonstrates a series of loading surfaces constructed for Magnezium AZ31 alloy in uniaxial tension. Electric impulses of different current densities $J_{m}\left(30,45,60\right.$, and $\left.70 \mathrm{~A} / \mathrm{mm}^{2}\right)$ were switched on during plastic deforming at $\sigma=246 \mathrm{MPa}$. The model values of $\sigma_{C}$ in (4.13) were calculated at $C_{1}=0.0265\left(\mathrm{~mm}^{2} / \mathrm{A}\right)^{C_{2}}$ and $C_{2}=1.0$. Since during the experiment considered the temperature is constant, we take $W(T)=1$. As a result, good agreement with experimental data is obtained (Rusinko and Varga, 2018): Eq. (4.13) gives values $\sigma_{C}$ for $J_{m}$ as 194.4, 159.9, $128,86 \mathrm{MPa}$ vs. $196.8,184.5,130,100.7 \mathrm{MPa}$ in the experiment, which means the relative error of $1.2,13,1.4,14 \%$. The orientation of the boundary plane being at the endpoint of the stress vector before the $\mathrm{DC}$ impulse, i.e. the value of angle $\beta_{1}$ from (4.8), is

$$
\beta_{1}=\arccos \frac{\sigma_{S}}{\sigma}=42.8^{\circ}
$$

where $\sigma_{S}$ is taken $180 \mathrm{MPa}$ (Nguyen et al., 2016).

Due to the axial symmetry of the loading surface in uniaxial tension, it is enough to provide information about the angle $\beta_{1}$. The radii of circles for the directions $\beta>\left|\beta_{1}\right|$ are calculated by Eq. (4.13)

$$
\sigma_{S m}=\frac{\sigma_{S}}{\sqrt{1+C_{m}^{2}}}
$$

where $C_{m}(m=\overline{1,4})$ are from Eqs. (3.2) and (3.3). Formula (4.15) gives the following values for $\sigma_{S m}: 140.9,115.7,98.7$ and $80.9 \mathrm{MPa}$.
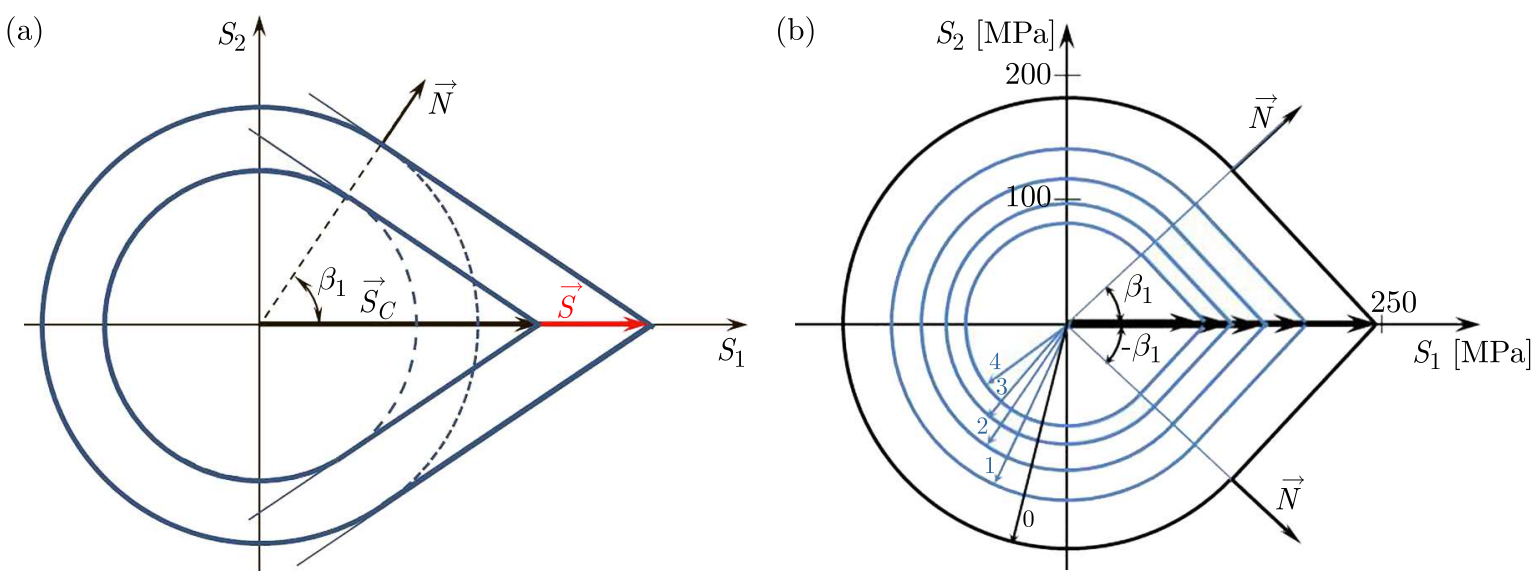

Fig. 4. (a) Loading surfaces corresponding to the stress drop effect. (b) Loading surfaces corresponding to the stress drop effect due to different values of current intensity. Numbers $1, \ldots, 4$ correspond to the indexes at $\sigma_{S m}$

\section{Creep deformation: ordinary case and deformation coupled with DC}

Since the duration of primary creep is usually much less than that of secondary creep, the time-integral in (4.3) can be ignored and we return again to Eq. (4.4) with the difference being that the rate integral is included in formula (2.4). As, after the active loading, the stress value remains 
unchangeable in time, the rate-integral decreases in the manner as shown below (Rusinko and Varga, 2018)

$$
H_{N}^{2}=\psi_{N}+I_{N}^{2}+S_{P}^{2}=\psi_{N}+[B \mathbf{S} \cdot \mathbf{N} \exp (-p t)]^{2}+S_{P}^{2} \quad S_{P}=\sqrt{\frac{2}{3}} \sigma_{P}
$$

The formula above states that the planes move toward the origin of coordinates with time, and the number of planes being on the endpoint of the stress vector increases. This process symbolizes the temporary increase in deformation. As $\exp (-p t) \rightarrow 0$, these movements terminate and we pass to the secondary portion of the creep with a constant rate. From the analysis of the rate integral (Rusinko and Rusinko, 2012) we can conclude that a) the constant $B$ governs the magnitude of primary creep - the grater $B$, the greater deformation develops in primary creep, b) the constant $p$ governs the duration of primary creep - the grater $p$, the faster the time-integral tends to zero, i.e. the shorter primary portion is. That is why we propose Eq. (3.4) when modelling the primary creep coupled with DC. The greater value of $B_{C}$, comparing to $B$, symbolizes the intensification of relaxation processes in the electric field during the steady state. And since the processes are quicker, they take less time to complete the primary creep - we introduce $p_{C}$ which is greater than $p$ by the current intensity.

Equations (3.1), (3.4) and (4.3) give

$$
\varphi_{N C}=\frac{2}{3 r}\left\{\sigma^{2}\left(1+C^{2}\right)(\cos \alpha \cos \beta \cos \lambda)^{2}-\left[\sigma B_{C} \exp \left(-p_{C} t\right) \cos \alpha \cos \beta \cos \lambda\right]^{2}-\sigma_{P}^{2}\right\}
$$

The strain intensity $\varphi_{N C}$ is non-zero in the following range

$$
\begin{aligned}
& \cos \lambda_{1 C}(t)=\frac{\cos \alpha_{1 C}(t)}{\cos \alpha} \quad \cos \alpha_{1 C}(t)=\frac{\cos \beta_{1 C}(t)}{\cos \beta} \\
& \cos \beta_{1 C}(t)=\frac{\sigma_{P}}{\sigma \sqrt{1+C^{2}-B_{C}^{2} \exp \left(-2 p_{C} t\right)}}
\end{aligned}
$$

By integrating the $\varphi_{N C}$ in Eq. (2.2) over range (5.3), we obtain the primary creep strain vector

$$
e(t)=a_{0} F\left[b_{C}(t)\right] \quad a_{0}=\frac{\pi \sigma_{P}^{2}}{9 r} \quad b_{C}(t)=\cos \beta_{1 C}(t)<b(t)
$$

where $b(t)$ is from Eq. (5.3) taken at $C=0$.

Since at the beginning of loading $(t=0)$ the rate-integral from (3.4) equals zero, the presence of DC results in the decrease of the radius of the initial sphere. Indeed, Eq. (3.1) at $\psi_{N}, I_{N}=0$ gives that

$$
H_{N} \equiv \sigma_{P C}=\frac{\sigma_{P}}{\sqrt{1+C^{2}}}
$$

Combining this fact with Eq. (5.1), we obtain Fig. 5a, where loading surfaces for $J=0$ and $J>0$ at a given instant of primary creep are shown. It unequivocally demonstrates the softening of the material under the action of current. Figure $5 \mathrm{~b}$ shows the dynamics of the change in boundary angles $\beta_{1 C}$ and $\beta_{1}$, which supports the sketch in Fig. 5a. One can see grater values of $\beta_{1 C}$ comparing to $\beta_{1}$ and their faster stabilization at a constant level. Figure $5 \mathrm{~b}$ is constructed on the base of the last relationship from (5.4) with constants taken from the authors' earlier work (Rusinko and Varga, 2018).

Formulae for the steady state creep rate are obtained from Eqs. (2.5) and (4.3), provided that $I_{N}=0$ and, as a result, $\dot{\psi}_{N}=0$. Therefore, Eq. (2.5) takes the following form

$$
H_{N}^{2}(t)=\psi_{N}(t)+S_{P}^{2}=\text { const }
$$



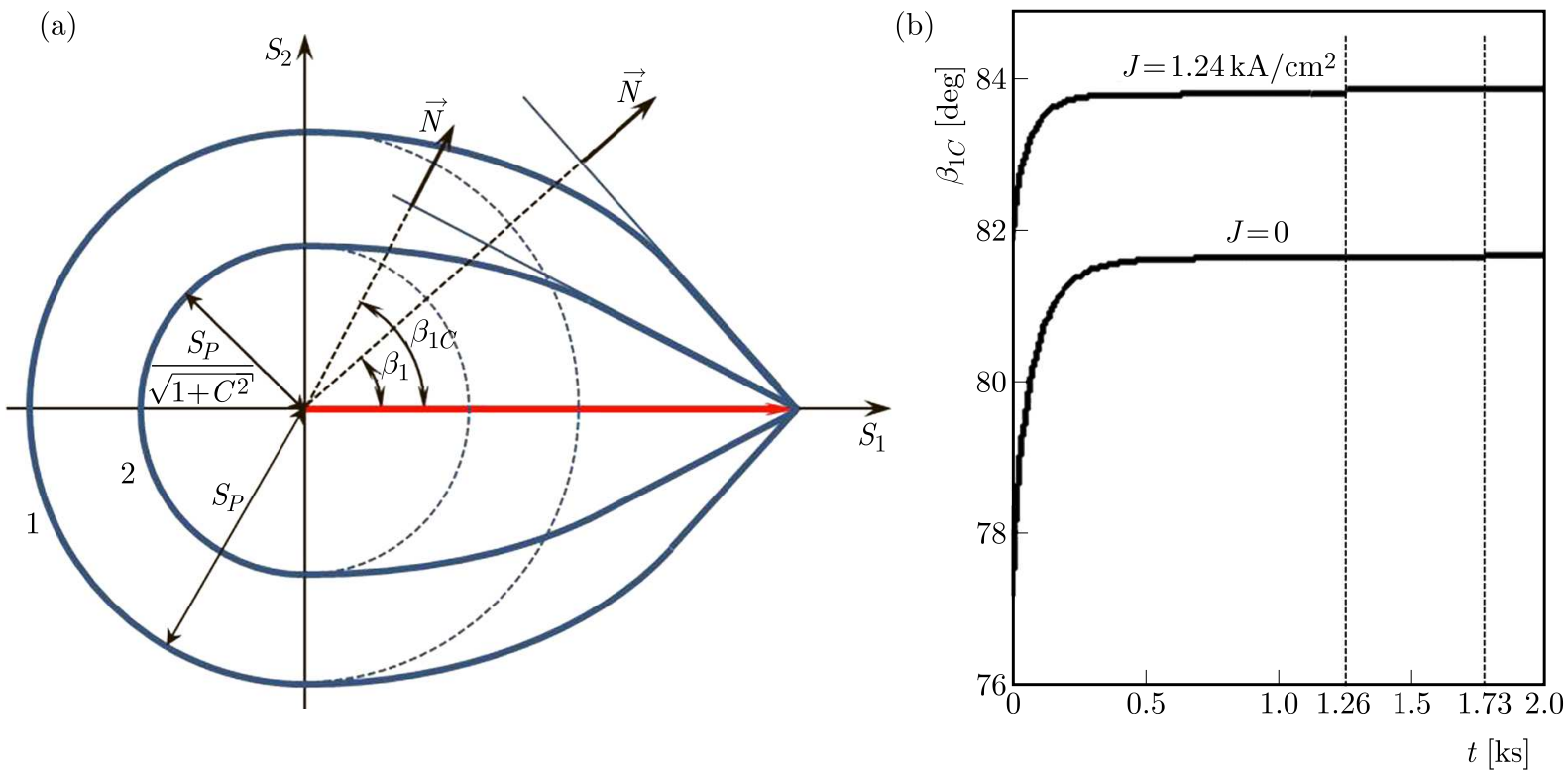

Fig. 5. (a) Loading surfaces at a given moment in the course of primary creep: $1-J=0,2-J>0$.

(b) Change in the boundary angles during primary creep of tin in uniaxial tension: $\sigma=4.63 \mathrm{MPa}$,

$$
T=348 \mathrm{~K}
$$

This means that the motions of planes occurring in the progress of primary creep terminate, and there is a set of planes on the endpoint of the stress vector which determines the value of the creep rate. The fact that $\dot{\psi}_{N}=0$ reflects equilibrium between the softening and hardening of the material during the secondary creep.

Equations (4.3) give the strain-intensity-rate in secondary creep as

$$
r \dot{\varphi}_{N}=K \psi_{N}=\text { const }
$$

If to introduce the term reflecting the action of DC upon the deformation, we get

$$
\dot{\varphi}_{N C}=\frac{K}{r} \psi_{N C}=\frac{2 K}{3 r}\left[\sigma^{2}\left(1+C^{2}\right)(\cos \alpha \cos \beta \cos \lambda)^{2}-\sigma_{P}^{2}\right]
$$

The values of boundary angles can be obtained from (5.3) by letting $I_{N}=0$

$$
\begin{aligned}
\cos \lambda_{1 C} & =\frac{\sigma_{P}}{\sigma \sqrt{1+C^{2}} \cos \alpha \cos \beta} \quad \cos \alpha_{1 C}=\frac{\sigma_{P}}{\sigma \sqrt{1+C^{2}} \cos \beta} \\
\cos \beta_{1 C} & =\frac{\sigma_{P}}{\sigma \sqrt{1+C^{2}}}
\end{aligned}
$$

Again, the final procedure is to calculate the macrodeformation via Eq. (2.2), which gives the secondary creep vector rate as

$$
\dot{e}_{C}=A F\left(b_{C}\right) \quad b_{C}=\cos \beta_{1 C} \quad A=\frac{\sqrt{3} c \sigma_{P}^{2}}{2 \sqrt{2}} \sigma^{k-2} K
$$

where $K$ is from Eqs. (2.7) and (3.5).

\section{Discussion}

By letting in (5.8) $\psi_{N C}=0$, we again arrive at result (5.5). This fact means that the electric field decreases the stress needed to induce irrecoverable straining. Further, inequality $\beta_{1 C}>\beta_{1}$, obtained by comparing (5.9) and (4.7), indicates that the number of slip systems involved in 
creep deformation increases due to DC. Furthermore, comparing to Eq. (4.5), the term $\left(1+C^{2}\right)$ in (5.8) shows more intensive development of creep deformation within every slip system. This fact is evident from Fig. 7a, where on the base of Eq. (5.6) and (5.9), the loading surfaces for ordinary secondary creep and that coupled with DC are shown. Consider two identically oriented planes 1 and 2, they are tangent to the sphere of radius from (5.5) and $S_{P}$ (ordinary creep), respectively. It is easy to see that for a given stress vector, when both planes are in position 3 , the distances traveled by the planes are different. If, for example, to follow the intersections of the planes with $S_{1}$-axis, it is obvious that $A A_{1 C}>A A_{1}$. This means that the distance traveled by plane in the presence of DC is greater than in ordinary creep. Figures $6 \mathrm{a}$ and $6 \mathrm{~b}$ demonstrate the change in $\sigma_{P C}$ and $\beta_{1 C}$ for tin as a function of current intensity and temperature.
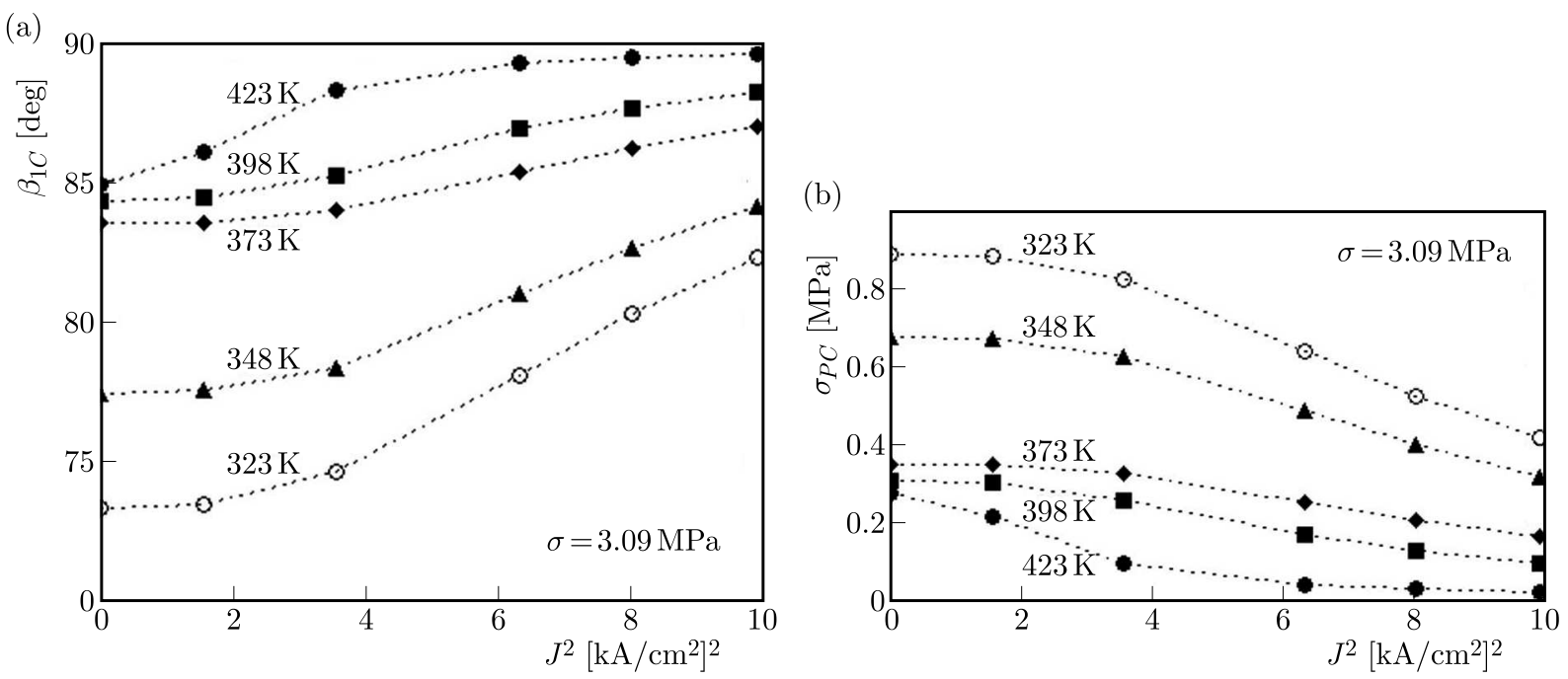

Fig. 6. (a) $\beta_{1 C} \sim J^{2}$ and (b) $\sigma_{P C} \sim J^{2}$ plots at different temperatures
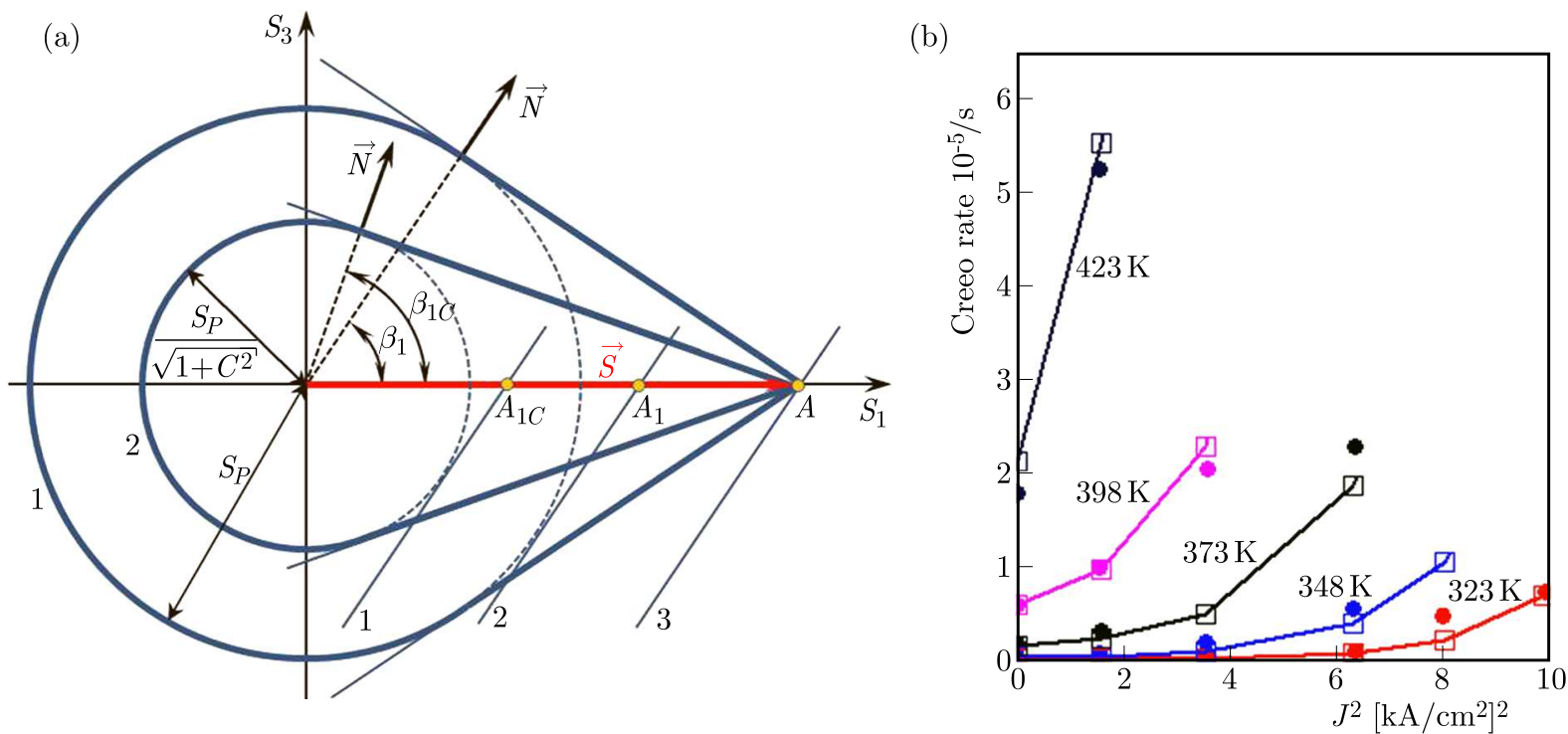

Fig. 7. (a) Loading surfaces for steady state creep: $1-J=0,2-J>0$. (b) $\sigma_{P C} \sim J^{2}$ plots at different temperatures: points - experiment, lines - model

The most important result is the dependence between the steady state creep rate and the current intensity at different temperatures, Fig. 7b. It must be stressed that, in contrast to our earlier works, Fig. 7b shows good agreement with experimental data not only for one temperature, but for the range from 323 to $423 \mathrm{~K}$. Graphs from Figs. 6 and 7 are constructed by Eqs. 
(3.2), (3.3), (5.5), (5.9) and (5.10) at the following values of model constants: $k=6, c=26$, $u_{1}=0.5\left(\mathrm{~cm}^{2} / \mathrm{kA}\right)^{u_{2}}, u_{2}=3.0, w_{1}=0.012 \mathrm{~K}^{-1}, w_{2}=4.102, w_{3}=6.0, w_{4}=0.12$.

\section{Conclusion}

Relationships to evaluate deformation properties of metals in electric fields are presented in the framework of the synthetic theory. In contrast to our earlier works, the results obtained for the steady state creep lead to good agreement with experiments for a wide range of temperatures. On the base of the relationships derived in terms of the synthetic theory, we have conducted a thorough analysis of loading surfaces for different cases of irrecoverable deformation coupled with direct current - plastic deformation and primary/secondary creep. The results obtained, especially their visualization in the form of loading surfaces, give valuable information for better understanding of the deformation properties of metals deformed in the electric field.

\section{References}

1. Andrawes J., Kronenberger T., Perkins T., Roth J.T., Warley J., 2007, Effects of DC current on the mechanical behavior of AlMg1SiCu, Materials and Manufacturing Processes, 22, $91-101$

2. Batdorf S., Budiansky B., 1949, Mathematical theory of plasticity based on the concept of slip, NACA, Technical Note, $\mathbf{8 7 1}$

3. Chen R., Yang F., 2008, Impression creep of a Sn60Pb40 alloy: the effect of electric current, Journal of Physics D: Applied Physics, 41, 155406

4. Kinney C., Morris J.W., Lee T.K., Liu K.C., Xue J., Towne D., 2009, The influence of an imposed current on the creep of Sn-Ag-Cu solder, Journal of Electronic Materials, 38, 221-226

5. Li W.-Y., Zhou M.-B., Zhang X.-P., 2015, Creep behavior of Cu/Sn-3.0Ag-0.5Cu/Cu solder joints under tensile stress coupled with DC current stressing, 16th International Conference on Electronic Packaging Technology (ICEPT), 11-14 Aug. 2015, Changsha, China, 187-192

6. Nguyen T.T., Nguyen T.V., Hong S.-T., Kim M.-J., Han H.N., Morestin F., 2016, The effect of short duration electric current on the quasi-static tensile behavior of magnesium AZ31 alloy, Advances in Materials Science and Engineering, p. 10, DOI: 10.1155/2016/9560413

7. Perkins T.A., Kronenberger T.J., Roth J.T., 2007, Metallic forging using electrical flow as an alternative to warm/hot working, Journal of Manufacturing Science and Engineering, 129, 84-94

8. Ross C.D., Irvin D.B., Roth J.T., 2007, Manufacturing aspects relating to the effects of direct current on the tensile properties of metals, Journal of Engineering Materials and Technology, 129, $342-347$

9. Rusinko A., 2016, Modeling the effect of DC on the creep of metals in terms of the synthetic theory of irrecoverable deformation, Mechanics of Materials, 93, 163-167

10. Rusinko A., Rusinko K., 2009, Synthetic theory of irreversible deformation in the context of fundamental bases of plasticity, Mechanics of Materials, 41, 106-120

11. Rusinko A., Rusinko K., 2011, Plasticity and Creep of Metals, Springer Berlin Heidelberg

12. Rusinko A., Varga P., 2018, Modelling of the plastic deformation and primary creep of metals coupled with DC in terms of the synthetic theory of irrecoverable deformation, Mechanics of Time-Dependent Materials, 23, 23-33

13. Sanders JR. J.L., 1954, Plastic stress-strain relations based on linear loading functions, Proceedings of the Second USA National Congress of Applied Mechanics, Ann Arbor, 14-18 June, $455-460$ 
14. Sanmartin A., KleinstüCK K., Quyen N.H., Paufler P., 1983, Influence of a direct current and a temperature gradient on the creep rate in V3Si, Physica Status Solidi A, 80, 2, K171-K174, DOI: $10.1002 /$ pssa.2210800250

15. VArga P., Rusinko A., 2018, Modeling the effects of imposed current on the creep of SAC305 solder material, 19th International Conference on Thermal, Mechanical and Multi-Physics Simulation and Experiments in Microelectronics and Microsystems (EuroSimE), 1-4

16. YANG F., Zhao G., 2010, Effect of electric current on nanoindentation of copper, Nanoscience and Nanotechnology Letters, 2, 322-326

17. Zhao G., Liu M., YAng F., 2012, The effect of an electric current on the nanoindentation behavior of tin, Acta Materialia, 60, 3773-3782

18. Zhao G., YAng F., 2014, Effect of DC current on tensile creep of pure tin, Materials Science and Engineering: A, 591, 97-104

19. Zhao K., FAn R., Wang L.J., 2016, The effect of electric current and strain rate on serrated flow of sheet aluminum alloy 5754, Journal of Materials Engineering and Performance, 25, 781-789 